Review

\title{
The Equipment of Czech Firefighters for the Detection and Field Analyses of Chemical Warfare Agents
}

\author{
Jana Krykorkova and Tomas Capoun * \\ Population Protection Institute, Na Luzci 204, Lazne Bohdanec 533 41, Czech Republic; \\ E-Mail: jana.krykorkova@ioolb.izscr.cz \\ * Author to whom correspondence should be addressed; E-Mail: tomas.capoun@ioolb.izscr.cz; \\ Tel.: +420-950-580-330; Fax: +420-950-580-101.
}

Received: 25 March 2014; in revised form: 19 May 2014 / Accepted: 26 May 2014 /

Published: 30 May 2014

\begin{abstract}
This paper describes the requirements for the devices of detection, chemical reconnaissance and field analyses of chemical warfare agents (CWA) and divides them into simple devices of detection, universal detectors, selective analyzers, multi-component analyzers and mobile laboratories. It also describes the devices of detection available within the Fire and Rescue Service of the Czech Republic (FRS CR) and compares them with some prospective trends of further development.
\end{abstract}

Keywords: chemical warfare agent; chemical reconnaissance; field analysis; detection; identification; detector; analyzer

\section{Introduction}

Chemical reconnaissance is one of the significant chemical countermeasures in the case of an emergency event such as an uncontrolled leak of chemical warfare agents (CWA), or their military or terrorist attack. For the decision-making process of state authorities, crisis staff and intervention units need reliable data concerning the character, extent and level of the contamination of the atmosphere, territory, equipment, objects, persons, water and foodstuffs. Rapid detection and field analyses are important for minimizing the impact of the event because it takes a long time to carry sample specimens to a laboratory for analysis [1-3]. Necessary information can be obtained at the place of an accident only by an appropriately organized system of chemical reconnaissance and field analyses using modern devices, appliances and corresponding methodical procedures. This paper focuses on the 
devices of detection and field analyses of CWAs used by the units of the Fire and Rescue Service of the Czech Republic (FRS CR).

There is a wide range of approaches for the categorization of types of chemical reconnaissance. In terms of evaluating the response of the device and the type of output signal, the devices are classified as simple detection apparatus, universal detectors, and analyzers. The analyzers are divided into two groups: selective analyzers and multi-component analyzers [4]. In addition to the above-mentioned groups of devices of detection for chemical reconnaissance and field analyses, there are also portable and mobile chemical laboratories. They are designed for more detailed and more varied analyses in the field including the preparation of samples for the analysis. The spectrum of performed analyses, laboratory operations and sample processing relies on proper equipment of those laboratories with instruments, detectors and analyzers.

\section{Specific Requirements for Detection and Field Analyses of CWAs}

Generally, it is possible to establish that all the above-mentioned groups of appliances can be utilized for CWA analyses. However, not all of them are appropriate, accordingly it is important to keep in mind the following specifications of the CWA detection apparatus [4,5].

High toxicity of CWAs requires detection at very low concentrations, which therefore requires appliances with a low limit of detection (LOD). CWAs are active at very low concentrations; nevertheless, there might be a wide range of other substances in the surrounding space, from exhaust gases of vehicles to various products of burning and explosions, as well as many other substances. The aim is to eliminate false or "overlapping" signals, which requires appliances with high selectivity. The high risks resulting from the leaks or misuse of CWAs impose high demands on the reliability of chemical reconnaissance. Therefore, exact and accurate measurements are crucial. The effectiveness of measurements for maximal limitation of casualties or afflicted persons as a result of the CWA leak or misuse is significantly dependent on the speed of their implementation. Therefore, during chemical reconnaissance, devices or appliances with a rapid response have to be applied. Chemical reconnaissance is carried out in a contaminated environment, which causes contamination of the detection apparatus and so requires the use of disposable equipment or devices that can be decontaminated. Chemical reconnaissance is often performed under very complex conditions in a demanding environment and in chemical protection suits, and, therefore, the applied devices or appliances should be simple and ergonomically undemanding.

\section{Simple Detection Apparatus}

Simple detection apparatus represent the standard and customary group of devices of detection. They are designed for rapid and simple measurements in the field and are mostly based on a colored chemical reaction of a detected substance with an appropriate reagent that is laid on a specific carrier. The colored result of the reaction is evaluated visually. Individual types of devices of detection differ by the reagent carrier, whereas the most common simple detection apparatus are those with an air-intake system and a detection tube where the reagent produces a color reaction with a specific agent laid on a sorbent [6]. Besides this, apparatus with the reagent on paper, chalk, fabric, etc., may also be used. 
Units of the FRS CR use three simple detection devices: the CHP-71 detection tubes pump (Figure 1) is a light, portable pump designed for continuous drawing of air through the detection tubes for CWAs: TT-11 (for nerve-agent), TT-12 (for hydrogen cyanide, cyanogen chloride, phosgene and diphosgene) and TT-15 (for mustard gas) [7]. DETEHIT nerve-agent detection strips are an example of a detector in which the agent is applied to fabric. It is intended for very sensitive detection of nerve-agents or other inhibitors of cholinesterase in the air or water and on surfaces [7]. The CALID-3 series of liquid CWA detector papers are designed for detecting and differentiating the three major groups of CWA in liquid form [7].

Figure 1. CHP-71 detection tubes pump.

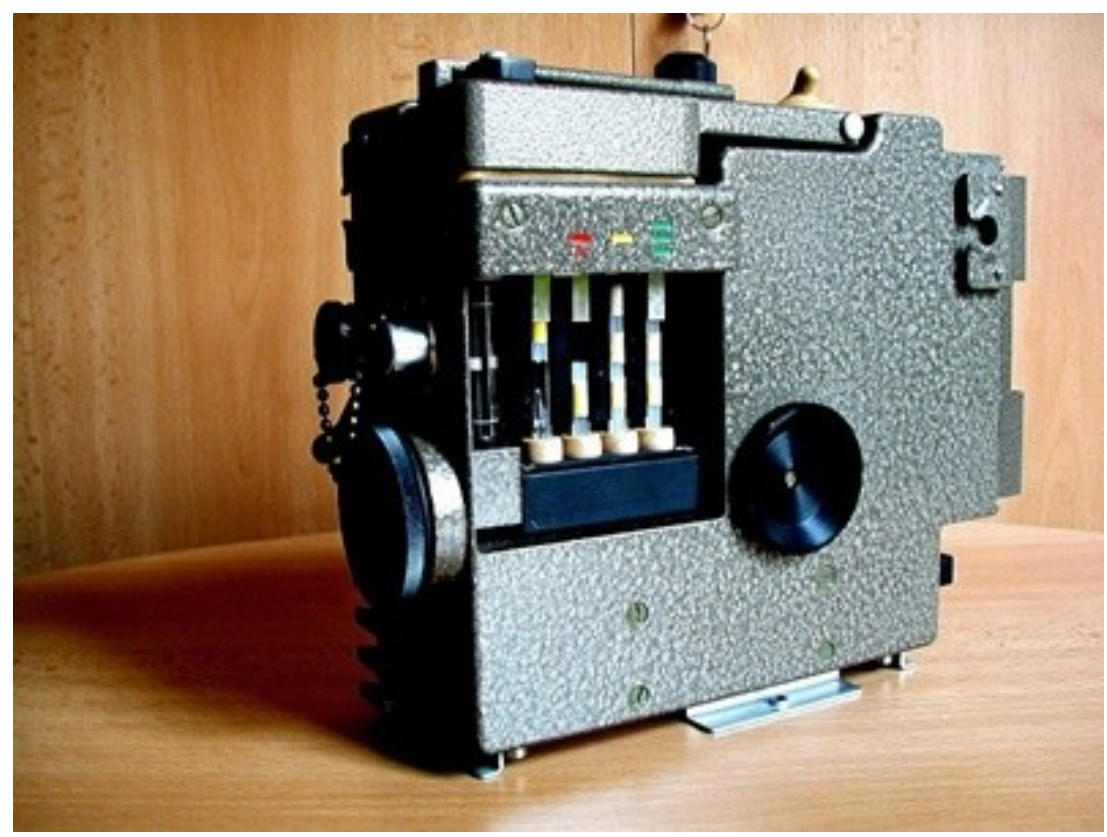

The main advantages of simple devices of detection applied in the place of an incident are the speed of measurements, the possibility of making an immediate decision, relatively low cost, small size and weight, easy maintenance and the fact that they do not require a specially qualified operator. The disadvantages are low selectivity, relatively low service life, subjectivity of the evaluation and low accuracy in the determination of CWAs.

\section{Universal Detectors}

These appliances perform the measuring on a particular general principle (e.g., the ability to ionize molecules by UV radiation), with which can be detected a greater number of substances. A number of principles can be applied for their construction, but in reality catharometers, semiconductor metal oxide sensors, and photoionization and flame ionization detectors prevail.

Universal detectors can be principally used for the determination of CWAs in the air, because of their rapid response, high reliability, robustness and also the possibility of decontamination. However, their practical use is impeded due to two basic properties, i.e., insufficient sensitivity and selectivity. Regarding sensitivity, it is possible to imagine searching for the source of a CWA leak using one of the mentioned universal detectors, the photoionization detector ppbRAE, which measures concentrations 
in ppb units. It shows high sensitivity to bis(2-chlorethyl)-sulphide (mustard gas) and nerve-agent type G, but lower sensitivity to agent VX [4]. Nevertheless, the importance of individual universal detectors for the detection of CWAs is minimal.

\section{Analyzers}

\subsection{Selective Analyzers}

Selective analyzers measure or monitor the concentration of certain dangerous substances, even in circumstances where the substance is in the atmosphere mixed with other gases and vapors. They are selective for the substance for which they are set and calibrated. However, it is not possible to perform identification of a completely unknown substance. The requirement for a selective measurement of a certain pre-set substance is a property or characteristic that is different from other analyzers. There are a large number of principles for the construction of selective analyzers including the electrochemical sensors, semiconductor sensors, infrared and UV analyzers and sensors, optoelectronic analyzers and photoacoustic analyzers [4].

The appliances with electrochemical sensors are based on measurements of changes of electrochemical potential and are an example of selective analyzers. Units of the FRS CR use detectors of various types and designs. They are mostly constructed as variable detectors with changeable electrochemical sensors. Sensors for certain CWAs are also available.

Semiconductor sensors are based on the measurement of the change of impedance or other characteristics of a semiconductor as a result of an exothermic, endothermic or other reaction on its surface. For selective detection of chemical agents, semiconductor calorimetric sensors measure heat released during a controlled catalytic chemical reaction between the sensor and the gas, this heat is proportionate to the concentration of measured gas are perspective. Intensive development of semiconductor selective sensors for toxic substances has taken place with the direct participation of the FRS CR in the Czech Republic. Their practical application in practice is appropriate due to the extremely low costs, possibility of miniaturizing to a millimeter scale, possibility of wireless transfer of the signal, long-term reliability and rapid response.

Infrared and UV analyzers are based on absorption in the infrared or UV part of the spectrum. They are mostly constructed such that the gas is sucked into a gas-cell where its spectrum is measured in a defined wavelength region and the appliance calculates the substance content in the air according to the absorption band intensity. These appliances are produced as single-gas analyzers or with calibrations selectable for several gases. An infrared analyzer, MIRAN, which is able to detect more than a hundred gases and vapors, is an example of an appliance used by the FRS CR. These multi-gas appliances can also be calibrated to CWAs.

Some units of the FRS CR are equipped with the analyzers based on an optoelectronic principle such as a chip measurement system, e.g., Dräger CMS. Essentially it works on the same principle as detection tubes, i.e., a chemical reaction resulting in a cultured product; however, it utilizes the objective optoelectronic evaluation of the discoloration. The evaluation of concentration values occurs automatically and is visualized on a display in ppm units. Regarding CWAs, chips for hydrogen cyanide and phosgene are available. 
Photoacoustic analyzers use a photoacoustic phenomenon based on the absorption of light of a specific frequency that heats the measured gas and increases the pressure with subsequent generation of an acoustic signal that is detected by microphones. Selectivity is therefore set by the wavelength of the light and the appliance can be calibrated to CWAs [5,7]. The Innova 1412 photoacoustic field gas-monitor [7] is an example of an analyzer used within the FRS CR.

Of all the selective gas analyzers, the multi-detectors are used most frequently. They enable simultaneous measurement of multiple gases, and are based on the combination of a universal sensor of flammable gases (catharometer, semiconductor sensor, photoionization detector) and changeable electrochemical sensors for toxic substances, e.g., an infrared sensor for carbon dioxide. The most common designs of multi-detectors simultaneously measure the concentrations of four to five different gases. The FRS CR mostly uses the RAE Systems, Dräger, Oldham and GasAlert apparatus. For the detection of CWAs, the sensors for hydrogen cyanide and phosgene are available.

\subsection{Multi-Component Analyzers}

The "gold standard" mobile and portable instrumental equipment is represented by multi-component analyzers, which-besides assessment of the concentration and long-term monitoring of harmful substances - enable identification of substances. The principle of identification is the measurement of specific characteristics of the substance, software comparison with the characteristics of substances stored in the appliance library, and identifying the substance with the most similar characteristics. They are high-quality analytical appliances whose work outside the laboratory no one imagined in the past. Nowadays, they are commonly constructed in a portable or mobile version to withstand shocks and fluctuations in weather and therefore they are suitable for chemical reconnaissance tasks. Units of the FRS CR use five types of analyzers: the ion-mobility spectrometers, detector array portable analyzers, portable gas chromatographs, infrared gas analyzers and mobile gas chromatographs with a mass detector.

Ion-mobility spectrometers, due to their extraordinary sensitivity are applied mainly for the analyses of CWAs where they are the most frequent devices of detection used within military and fire rescue units [5,7-9]. They are based on ionization of a molecule of the substance by a $\beta$-emitter and separation of generated ions on the basis of different mobilities. The result is a spectrum with peaks whose positions are characterized by the ion's relative mobility and which determines the kind of substance, and the intensity of the peak determines the CWAs concentration in the analyzed air. At the FRS CR, a Rapid Alarm Identification Device (RAID) series of CWA detectors [7,10] is commonly used.

An example of a portable analyzer using the principle of detector array — of which there are about twenty within the units of the FRS CR - is the Portable Detector of Dangerous Gases and Chemical Warfare Agents GDA-2 [11] (Figure 2). It represents a combination of selective principles (ion-mobility spectrometry and electrochemical principle) with universal detection by semiconductor sensors (for all flammable gases and vapors) and photoionization (for all gases and vapors with photoionization potential lower than the energy of an UV lamp). The concentration of a dangerous substance in the air is characterized by a certain signal in some of the mentioned sensors. The software of the appliance evaluates the proportion of the signals on individual sub-detectors, compares them with the data stored 
in the library and assigns the most probable chemical substance; it can also identify simple mixtures of two to three substances. Forty to fifty substances are stored in the appliance library including nerve-agents (tabun, sarin, soman, cyclosarin, VX), blistering agents (sulphide and nitrogen yperite and lewisite) and further asphyxiating and generally toxic substances.

Figure 2. The GDA-2 portable detector of dangerous gases and chemical warfare agents.

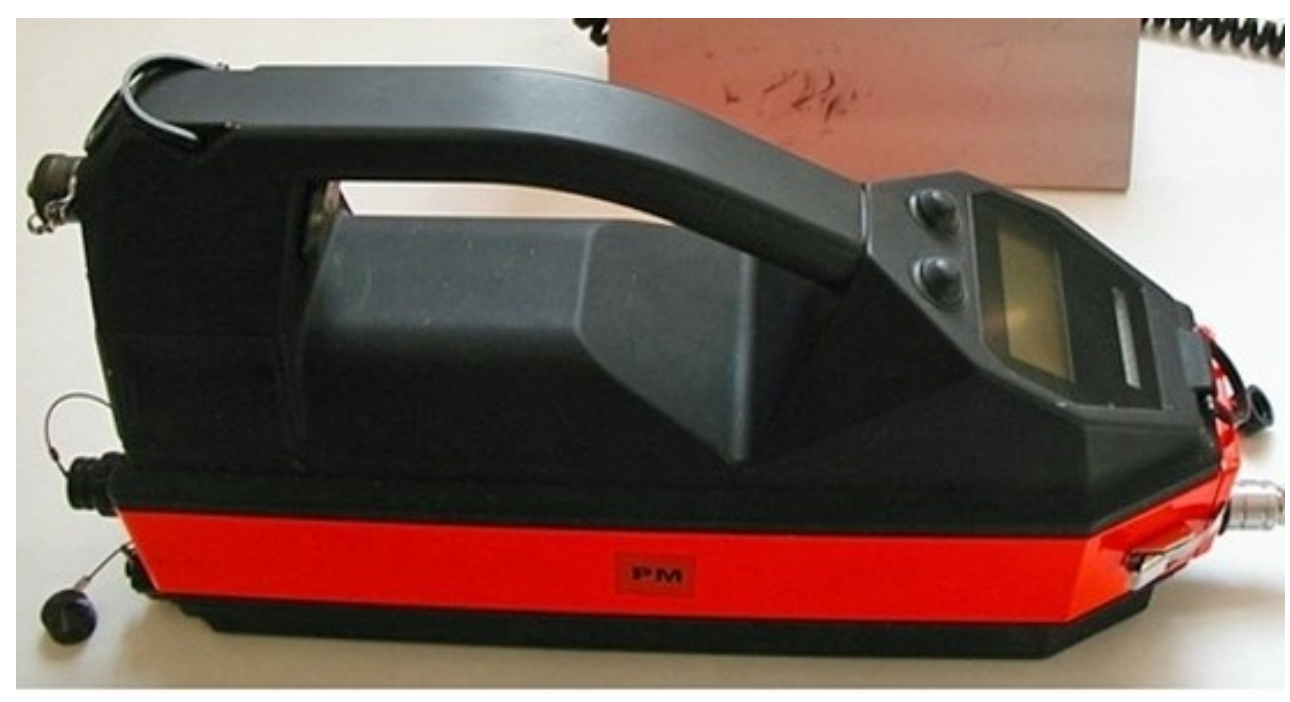

Portable gas chromatographs are designed for automatic identification and determination of gas and vapor concentration in air [5]. Chromatograph analysis mostly occurs on various capillary columns and the analysis output is a chromatogram that includes an overview of the peaks with retention times and a summary of names of identified substances with concentration values. Some units of the FRS CR use the Voyager portable digital gas chromatograph.

Infrared analyzers of gases are in principle the current FTIR spectrometers that, after measuring an infrared spectrum, carry out a comparison of the measured data with the spectra stored in the library and evaluate the most similar spectra of substances according to their calculated probability. Their most significant advantage is the high speed of analysis.

Mobile chemical laboratories of the FRS CR are equipped with Gasmet DX 4010 multi-gas FTIR analyzers [12] with a 10-m light-path gas-cell that is capable of identifying up to thirty gases and vapors of unknown composition in a mixture. The transportable GC/MS system EM 640 [7] represents the most sophisticated type of a specified class of analyzers that interconnect the separation of dangerous substances with the application of the gas-chromatography principle and their identification by mass spectrometry. The applications are basically identical to stationary devices.

Due to the high toxicity of CWAs and the associated risks during the measurements, the FRS CR also uses the principle of remote detection. A monitoring vehicle (Figure 3) with a remote detector SIGIS 2 (Scanning Infrared Gas Imaging System) is available for the FRS CR. This apparatus is designed for identification of clouds of toxic substances and CWAs from large distances from the place of the spills (up to $5 \mathrm{~km}$ ). It is a passive infrared detector with Fourier transformation (FTIR) that uses natural radiation of the surrounding areas and therefore is not equipped with its own source of radiation [13]. The remote detector SIGIS 2 is similar to the M21 Remote Sensing Chemical Agent Alarm (RSCAAL) that is used in the USA [14]. 
Figure 3. Monitoring vehicle with remote detector SIGIS 2.

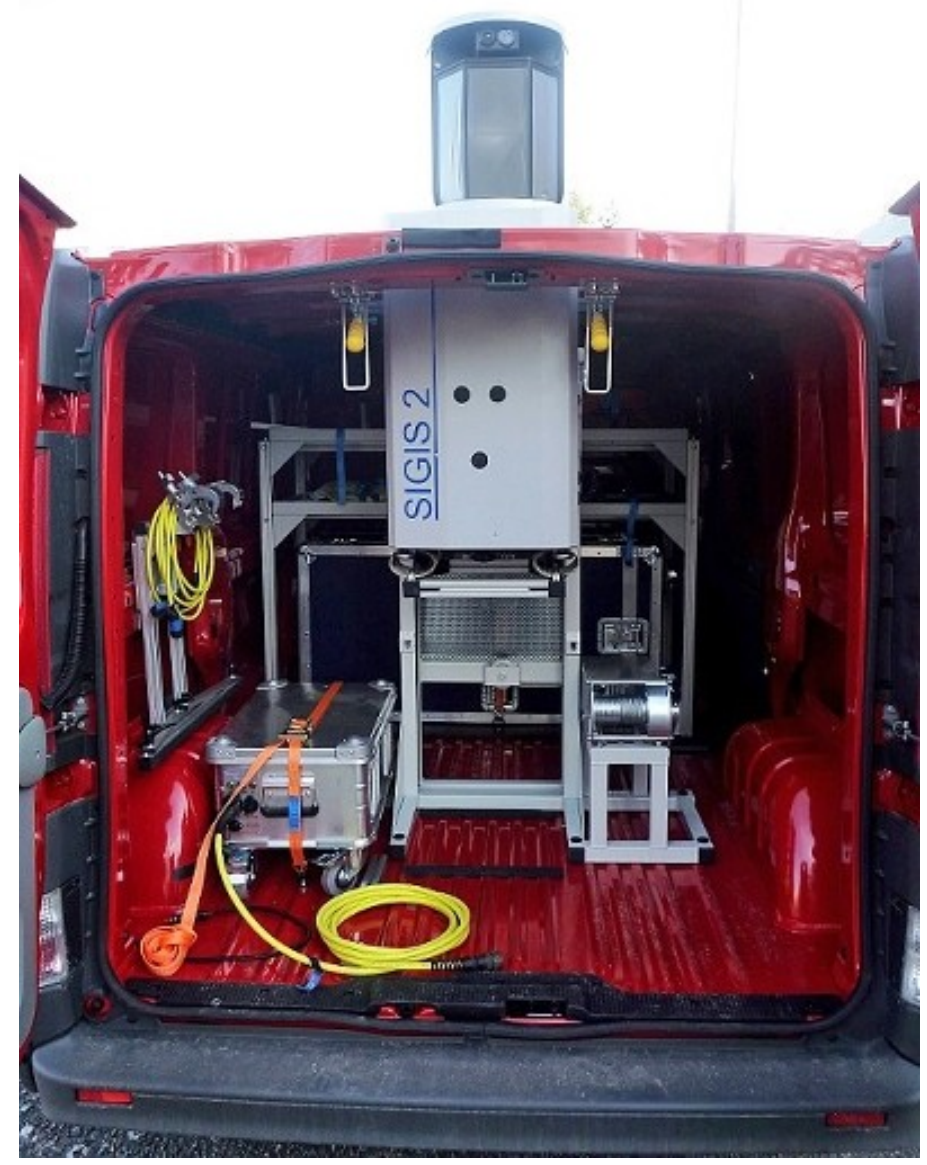

The above-mentioned overview of appliances focuses mainly on gases and vapors because the inhalation intoxication represents the most serious aspect during emergency events [5]. For comprehensiveness it should be added that during the last decade the technology and techniques also underwent rapid development in the area of a field analysis of liquids and solids.

The analyzers used by the FRS CR can be rated among these appliances. The portable Raman spectrometer FirstDefender (Figure 4) and the portable FTIR spectrometer TruDefender are designed for identification of a wide spectrum of organic and inorganic liquids and solids including their mixtures. They contain libraries of toxic industrial pollutants, medicines, pharmaceutical chemicals, explosives, drugs, pesticides, oil products, plastics, organic and inorganic fertilizers, various household commercial products and "white powders". The library of CWAs is an obvious component. A suitable addition to both appliances is the portable FTIR spectrometer TruDefender FTG, which is used for identification of unknown gases and vapors over solid and liquid samples (head-space method), which decreases the risk of potential inhalation intoxication or an explosion during the manipulation with unknown substances. Providing this set of appliances is complete with the devices of determination of an elemental composition of substances (the FRS CR uses, for example, a hand-operated ED X-ray spectrometer, Alpha-4000 S) then the success of field identification of unknown substances is higher than $90 \%$ [4]. 
Figure 4. Portable Raman spectrometer FirstDefender.

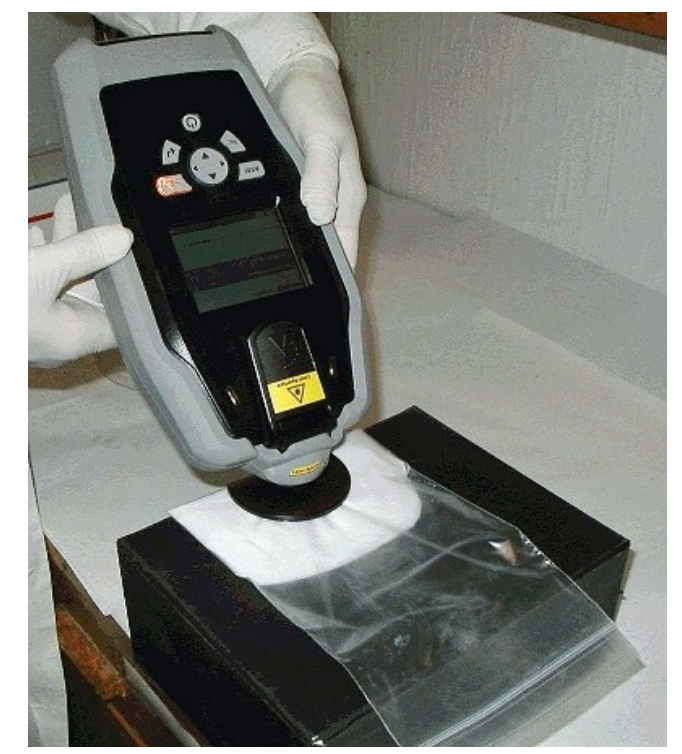

\section{Mobile Chemical Laboratories}

For the purposes of field analytical chemistry, the market nowadays offers a wide range of various products and analytical sets. Portable photometers, $\mathrm{pH}$ meters, titrators, etc., can also be used for field analyses of CWAs and are regular parts of modern sets [4].

In order to carry out analytical studies directly at the place of an incident — while in contact with the firefighting unit — and to bring frequently difficult conditions of a field analysis closer to the conditions in stationary laboratories, mobile chemical laboratories have become more and more significant [4]. They are sets of appliances, detection devices, analyzers, aids and reagents stored in a suitable vehicle body. Their main importance lies in their ability to perform a wide spectrum of measurements, as well as physical, physicochemical and analytical operations. The width of this spectrum depends on the quality of the device and the technical equipment.

Mobile chemical laboratories of the FRS CR (Figure 5) are constructed as special vehicles for intervention groups. They are designed for the detection, identification and determination of CWAs and other toxic substances in various samples of the environment. They also have high-quality mobile instrumentation to identify substances of unknown composition, e.g., a gas analyzer GDA-2, multi-gas FTIR analyzer Gasmet DX 4010, transportable GC/MS system EM 640, portable photometer, and Raman and FTIR spectrometers.

\section{Overall assessment of the Equipment of the FRS CR for the Detection and Field Analyses of CWAs}

The FRS units use various tools and instruments representing all directions of their development to perform detection and field analysis of the CWA. Development of these means of detection worldwide and in the Czech Republic continues on the appliances that so far have been exclusively the domain of stationary laboratories and has been aimed at their miniaturization, increasing their robustness and on the research of applications in the field. An example is the development of a new detector using the 
principle of mass spectrometry with counterflow chemical ionization (APCI-MS-CFI), published in 2006 in Japan $[5,15,16]$. This new detector is capable of detecting all CWAs including the lacrimal ones in concentrations in ten-thousandths of ppm in a few seconds. Furthermore, it is designed for the detection of explosives, drugs, medicaments, etc. Another developmental trend is the modernization of devices of monitoring for CWAs in the air based on a color reaction on an "infinite" tape or tab impregnated with specific reagents with spectrophotometric evaluation of color changes $[5,17]$.

Figure 5. Laboratory section of the mobile chemical laboratory of the Fire and Rescue Service of the Czech Republic (FRS CR).

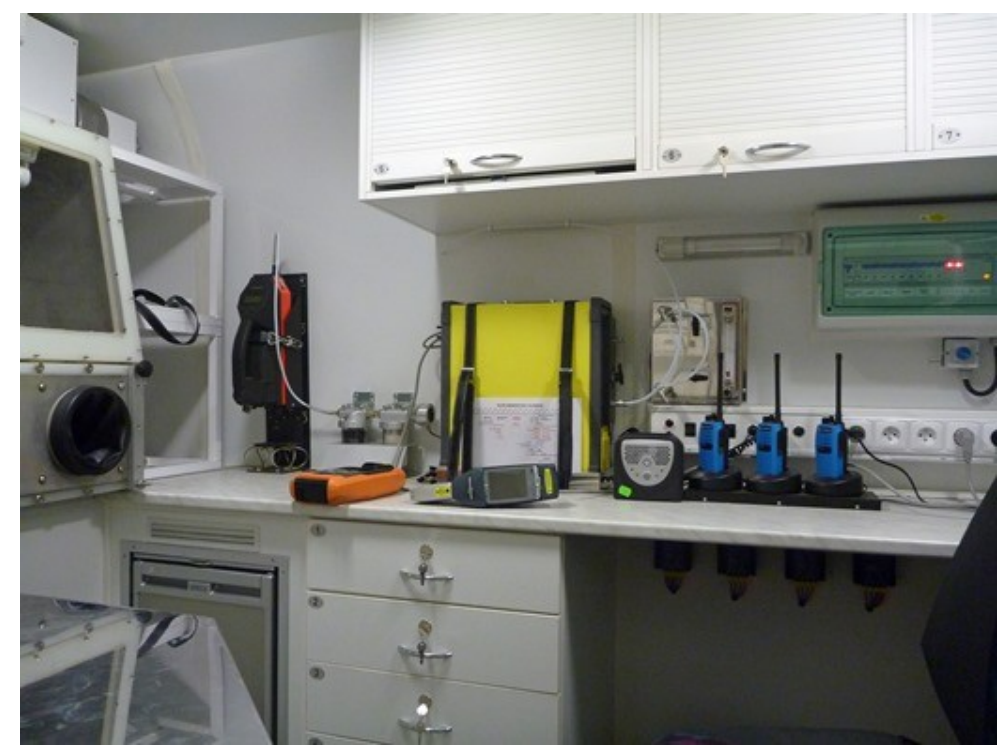

It is apparent that in the future there will be a large choice of devices. However, it will always be necessary to consider the purpose of usage of these devices, since not all devices of detection are usable in all circumstances. There is even an opinion that detection of CWAs may be resolved by the development of a portable version of ion-mobility spectrometers. These modern, fully automated devices - albeit intended for the analysis of substance leakage - measure only at low concentration values. They easily overload at the leakage or dispersion point of a substance therefore detect nothing and deactivate themselves automatically through perfusion, even for prolonged periods of time. Other user-friendly analyzers may experience problems with the background detection at the beginning of a detection after CWA leakage.

Table 1, which compares properties and characteristics of selected detectors and analyzers, shows to what extent they satisfy some specific CWA detection requirements of the FRS CR (see Section 2). Preparation time is the period from switching the equipment on to the commencement of a measurement. In order to evaluate the sensitivity of the instruments, it is necessary to compare their limits of detection with their lethal concentration values $\left(\mathrm{LCt}_{50}, \mathrm{mg} \cdot \mathrm{min} / \mathrm{m}^{3}\right)$. This value indicates the vapor concentration leading to $50 \%$ death in humans with $1 \mathrm{~min}$ inhalation and amounts to $60 \mathrm{mg} \cdot \mathrm{min} / \mathrm{m}^{3}$ for soman, $40 \mathrm{mg} \cdot \mathrm{min} / \mathrm{m}^{3}$ for agent VX and $1500 \mathrm{mg} \cdot \mathrm{min} / \mathrm{m}^{3}$ for mustard gas [5]. The numerical data in the Table 1 were determined in the chemical laboratory of the FRS CR [4]. Air mixtures were prepared from real agents using the dynamic calibration unit Sycos K-DPG (Ansyco, Karlsruhe, Germany). 
Table 1. Determined limits of detection (LOD) of chemical warfare agents (CWAs) and analysis times of some detectors and analyzers introduced within the FRS CR.

\begin{tabular}{|c|c|c|c|c|c|}
\hline Detector (analyzer) & $\begin{array}{l}\text { Preparation } \\
\text { time, min }\end{array}$ & $\begin{array}{l}\text { Response } \\
\text { time, min }\end{array}$ & Soman & $\begin{array}{c}\text { LOD, } \mathbf{m g} / \mathrm{m}^{3} \\
\text { Agent VX }\end{array}$ & $\begin{array}{c}\text { Mustard } \\
\text { gas }\end{array}$ \\
\hline $\begin{array}{c}\text { CHP-71 detection tubes pump with detection tubes TT- } \\
11 \text { (for nerve-agent) and TT-15 (for mustard gas) }\end{array}$ & 2 & 4 & 0.5 & 1 & 5 \\
\hline DETEHIT nerve-agent detection strips & 0.5 & 3 & 0.02 & 0.1 & - \\
\hline Photoionization detector ppbRAE & 2 & 0.3 & 0.2 & 0.6 & 0.5 \\
\hline $\begin{array}{l}\text { Innova } 1412 \text { photoacoustic } \\
\text { field gas-monitor }\end{array}$ & 1 & 0.5 & 3 & 3 & 1 \\
\hline $\begin{array}{c}\text { Rapid alarm and identification } \\
\text { device RAID-1 }\end{array}$ & 15 & 0.5 & 0.03 & 0.05 & 1 \\
\hline Portable detector of dangerous gases and CWA GDA-2 & 2 & 0.5 & 0.01 & 0.02 & 0.5 \\
\hline Multi-gas FTIR analyzer Gasmet DX-4010 & 15 & 3 & 8 & 12 & 8 \\
\hline $\begin{array}{c}\text { Transportable GC/MS system EM } 640 \text { - air sampling } \\
\text { by tube Tenax }\end{array}$ & 30 & 20 & 0.07 & 0.02 & 0.02 \\
\hline $\begin{array}{l}\text { Transportable GC/MS system EM 640 - air sampling } \\
\text { by technique SPME (CAR/PDMS) }\end{array}$ & 30 & 20 & 5 & 2 & 2 \\
\hline
\end{tabular}

\section{Conclusions}

The overview of devices of chemical reconnaissance and field analyses for the purposes of the detection of CWAs available within the FRS CR demonstrates that these devices are clearly at the world-class and correspond with the tasks and missions of the FRS CR in emergency situations associated with the leakage or misuse of CWA substances. The comparison of appliances with regard to general requirements for the detectors of CWAs and with regard to the necessary sensitivity shows that, in the future, equipment replenishment of the FRS units will need to consider the purpose of the utilization of the devices of detection. It results in the required sensitivity, as well as suitable concentration ranges of measurements, specific substances, frequency of monitoring, and a way to transfer information.

\section{Acknowledgments}

This study was supported by the Ministry of Interior of the Czech Republic (project of security research and development VF20112015018).

\section{Authors Contributions}

Tomas Capoun performed the literature review and wrote the first version of the manuscript. Jana Krykorkova checked and amended the final version of the article. Both authors participated in the experimental work in the laboratory.

\section{Conflicts of Interest}

The authors declare no conflict of interest. 


\section{References}

1. Harris, C.M. The science of detecting terror. Anal. Chem. 2002, 74, A126-A133.

2. Smith, W.D. Analytical chemistry at the forefront of homeland defense. Anal. Chem. 2002, 74, A462-A466.

3. Fittch, J.P.; Raber, E.; Imbro, D.R. Technology challenge in responding to biological and chemical attacks in the civilian sector. Science 2003, 302, 1350-1354.

4. Capoun, T. Chemical Reconnaissance and Field Analysis. In Chemical Weapons and Protection against Them, 1st ed.; Pitschmann, V., Ed.; Manus: Prague, Czech Republic, 2011; pp. 34-63.

5. Seto, Y. On-Site Detection of Chemical Warfare Agents. In Handbook of Toxicology Chemical Warfare Agents, 1st ed.; Gupta, R.C., Ed.; Academic Press Elsevier: London, UK, 2009; pp. 813-825.

6. Pitschmann, V. Analysis of Toxic Agents through Detection Tubes, 2nd ed.; Econt Consulting: Drahelcice, Czech Republic, 2005.

7. Gibson, N.; Rovery, M. IHD Jane's EOD \& CBRNE Defence Equipment 2012-2013, 1st ed.; IHS Jane's, IHS Global Limited: Coulsdon, UK, 2012; pp. 261-337.

8. Cottingham, K. Ion mobility spectrometry rediscovered. Anal. Chem. 2003, 75, A435-A439.

9. Iceman, G.A.; Stone, J.A. Ion mobility spectrometers in national defense. Anal. Chem. 2004, 76, A390-A397.

10. Ion Mobility Spectrometry. Available online: http://www.bruker.com/products/cbrne-detection/ ims.html (accessed on 7 March 2014).

11. GDA-2. Available online: http://www.airsense.com/en/products/gda-2/ (accessed on 7 March 2014).

12. GASMET FTIR Gas Analyzers. Available online: http://gasmet.fi/products/ftir_gas_analyzers (accessed on 14 November 2013).

13. Harig, R. Passive remote sensing of pollutant clouds by FTIR spectrometry: Signal-to-noise ratio as a function of spectral resolution. Appl. Opt. 2004, 43, 4603-4610.

14. O’Hern, M.R.; Dashiell, T.R.; Tracy, M.F. Chemical Defense Equipment. In Medical Aspects of Chemical and Biological Warfare, Teil 2; Sidell, F.R., Takafuji, E.T., Franz, D.R., Eds.; The Office of the Surgeon General Department of the Army, Borden Institute: Washington, DC, USA, 1997; p. 381.

15. Takada, Y. Rapid detection of explosive residues. Bunseki 2006, 55, 328-332.

16. Seto, Y.; Kanamori-Kataoka, M.; Tsuge, K.; Ohsawa, I.; Iura, K.; Itoi, T.; Sekiguchi, H.; Matsushita, K.; Yamashiro, S.; Sano, Y.; et al. Sensitive monitoring of volatile chemical warfare agents in air by atmospheric pressure chemical ionization mass spectrometry with counter-flow introduction. Anal. Chem. 2013, 85, 2659-2666.

17. Nakano, N.; Nagashima, K. Development of tapes for the determination of toxic gases by a monitoring tape method. Bunseki 2001, 50, 7-14.

(C) 2014 by the authors; licensee MDPI, Basel, Switzerland. This article is an open access article distributed under the terms and conditions of the Creative Commons Attribution license (http://creativecommons.org/licenses/by/3.0/). 